\title{
Silver Stars
}

\section{Camilla Juul Hansen ${ }^{1}$ and Francesca Primas ${ }^{1}$}

${ }^{1}$ European Southern Observatory, Karl-Schwarzschild-Strasse 2, D-85748 Garching, Germany email: cjhansen@eso.org, fprimas@eso.org

\begin{abstract}
The rapid neutron-capture process (r-process), which produces some of the heaviest elements, is not well understood. Obtaining accurate abundances of these heavy elements $(Z>$ 38 ) is important, both in the context of the chemical evolution of the Galaxy and for understanding the site(s) and process(es) of formation of those elements. We have determined elemental abundances for several r-process elements, notably silver, from high resolution VLT/UVES spectra. Silver was chosen because it is predominantly a light r-process element $(38<\mathrm{Z}<50)$, and little is known about its formation and evolution in the Galaxy. Here, we present our preliminary results.
\end{abstract}

Keywords. Silver, abundances, r-process

\section{Introduction}

Recent observational studies (François et al. 2007, Montes et al. 2007) of neutroncapture elements in metal-poor stars have provided indications that the r-process has a second component (as is the case for the slow neutron-capture process (s-process) Travaglio et al. 2004). Of the heavier elements $(\mathrm{Z}>38)$, the lighter of these $(\mathrm{Z}=38$ $50)$ are seen to be over-abundant compared to the scaled solar abundances of elements with $\mathrm{Z}>50$ (such as Ba, La,... Eu etc.) at low metallicity, hence a second process (the so-called Lighter Element Primary Process - LEPP) has been proposed to explain this relative excess. In order to test the LEPP hypothesis, we chose to target silver (previously studied in only seven stars by Crawford et al. 1998), since the r-process dominates its production (79\%, Arlandini et al. 1999), making it a good tracer in the interval $38<\mathrm{Z}$ $<50$.

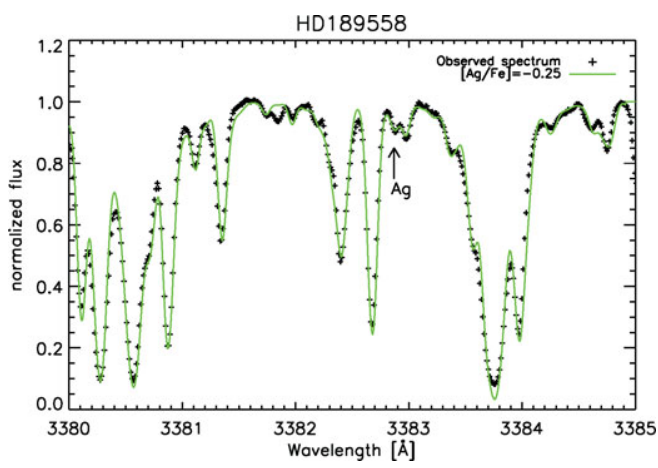

Figure 1. A best fit (solid line) of the Ag line at $3382 \AA$ in the star HD189558 (plusses).

\section{The Sample}

The sample consists of dwarf stars spanning a broad range in temperature (T: 5400 - 6500K), gravity (log g: $3.73-4.74$ dex) and metallicity $([\mathrm{Fe} / \mathrm{H}]:-0.54$ to -3.37$)$. All 
the stellar spectra have been reduced using the UVES pipeline and shifted, added, and normalized in IRAF. MARCS (Gustafsson et al. 2008) stellar model atmospheres and MOOG spectrum synthesis code were used in order to derive the abundances. In Figure 1, a fit to the silver line at $3382 \AA$ in HD189558 (a cool dwarf) is shown. Silver has two main resonance lines, both in the UV.

\section{Results and Future Work}

We have detected silver in 27 stars with metallicities down to $[\mathrm{Fe} / \mathrm{H}] \sim-1.55$. Silver absorption was looked for in metal-poor $([\mathrm{Fe} / \mathrm{H}] \sim-3.4)$ dwarfs, but no lines were detected, and several tests have shown that metal-poor giants are better suited for this study. Other elements such as Y, Zr, Ba and Eu have also been determined for our dwarf stars, in order to compare silver to different $r$ - and s-process elements (see preliminary results in Figure 2).
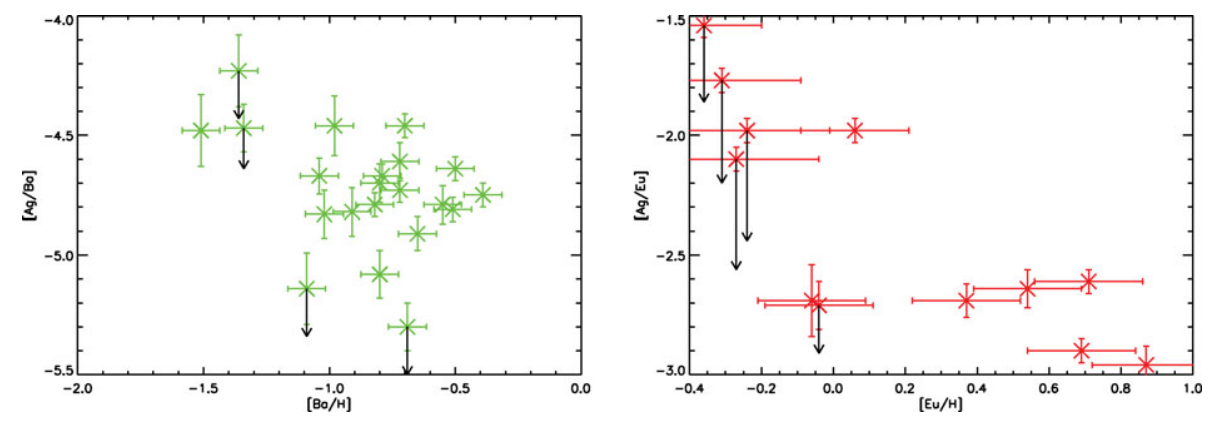

Figure 2. $[\mathrm{Ag} / \mathrm{Ba}]$ vs $[\mathrm{Ba} / \mathrm{H}]$ (left) and $[\mathrm{Ag} / \mathrm{Eu}]$ vs $[\mathrm{Eu} / \mathrm{H}]$ (right) abundance ratios and associated uncertainties. Arrows indicate abundance upper limits.

At metallicities of our sample $([\mathrm{Fe} / \mathrm{H}]>-1.6)$, it is not surprising that $\mathrm{Ag}$ shows a tighter correlation with Eu than with Ba (see Figure 2). Eu is a r-process element (94\% main r-process, Arlandini et al. 1999) while Ba is mainly produced by the s-process like other elements we have looked at (e.g. Zr).

At our stellar metallicities, it is not possible to characterize the LEPP and silver does not seem to be able to prove the existence of this process, the impact from the LEPP at $[\mathrm{Fe} / \mathrm{H}]>-1.6$ have been erased and contributions from other processes, such as the main $\mathrm{r}$ - and s-process, are seen instead.

Future work will explore metal-poor giants, thereby increasing the sample and hopefully obtaining silver at lower metallicities allowing us to trace and characterize the LEPP through determinations of key r- and s-process elements.

\section{References}

Arlandini et al. 1999, AJ,525, 866

Crawford et al. 1998, AJ, 116, 2489

François et al. 2007, A\& $A, 476,935$

Gustafsson et al. 2008, A\& $A, 486,951$

Montes et al. 2007, AJ, 671, 1685

Travaglio et al. 2004, AJ, 601, 864 